Article

\title{
Cartesian Material Space with Active-Passive Densities of Complex Charges and Yin-Yang Compensation of Energy Integrals
}

\author{
Igor Bulyzhenkov 1,2 (1D) \\ 1 Lebedev Physics Institute RAS, Moscow 119991, Russia; ibw@sci.lebedev.ru \\ 2 Moscow Institute of Physics \& Technology, Dolgoprudny 141700, Russia
}

Received: 9 April 2018; Accepted: 29 May 2018; Published: 5 June 2018

check for updates

\begin{abstract}
The non-empty space approach to Maxwell's equations reveals the analytical solution with the continuous radial density of the extended astroparticle. The principle of equivalence works universally for active/passive masses and for active/passive electric charges. The Maxwell equations for complex continuous charges become four-vector identities for locally-compensated flows of active (yang) and passive (yin) energies. The Einstein formula for relativistic mass-energy can be supplemented by the imaginary energy of electricity. Cartesian relativistic physics should have its own non-dual analog of the Einstein equation.
\end{abstract}

Keywords: material space; extended astroparticles; holism; imaginary charges; inside heat; conceptual experiments; yin-yang dialectics

\section{Introduction}

Cartesian physics [1,2] of material space is currently described only in qualitative terms and without quantitative predictions of specific phenomena for the theory verification/falsification procedure. This physics refers to Descartes's natural philosophy [3,4], which is denied by the dual Newton world with empty space between localized mechanical bodies. The concept of void space regions without matter was unclear to Descartes, who, after Aristotle, maintained the extension of matter or 'matter-extension' as the continuous material plenum. The Cartesian vortex mechanics for all types of observable spatial displacements in this material plenum had been inferred since 1629. Later, Newton's successful dynamics of point-like masses rejected the need for sophisticated matter-extensions with vortex states. Nowadays, Newton's mechanics of localized masses and continuous gravitation fields in empty space form the dual version of matter for the modern space-time theory for the Solar system and beyond. Not Cartesian physics, but Newtonian empty space modeling became mandatory for Einstein's 1916 gravitation in the weak field limit. At that time, no one looked at mechanical theories if they did not fit Newton's mass transport at low speeds.

By rejecting invisible material phenomena beyond the limits of laboratory measurements, it is impossible to understand, for instance, Cartesian natural philosophy, the yin-yang Chinese dialectics [5] and the Russian cosmism [6] principles ("people are citizens of the entire Universe rather than the Earth"). Beginning with Plato, the Ancient Greeks considered space to be a continuous material plenum, filled everywhere by 'the most translucent kind (Aether)'. Material bodies cannot be replaced, according to Aristotle's logical proof, with a completely empty, intangible place outside the continuous space-plenum. Descartes followed this logical avenue and eventually offered his vortex mechanics for the spatial transport of variable energy charges. The question is, should one neglect Cartesian physics of non-empty space due to 'the laboratory evidence' in favor of the Newtonian empty space model? 
Dual classical physics for spatially-separated matter and fields is traditionally assigned to the macroscopic world, while non-dual material densities of quantum fields physics is assigned only to the microscopic world. However, the unique physical reality is either dual or non-dual regardless of the spatial scaling and mathematical formalisms in available theories. Similarly, the matter is either local or non-local regardless of suitable approaches to describe it. The celebrated Einstein-Podolsky-Rosen paradox initiated the long-term discussion and many tests of the material world's nonlocality. Now, the nonlocality of matter is reasonably considered beyond quantum physics [7]. The world holism [8] and the global direct overlap of all material elements motivate our attempts to develop non-Newtonian mechanics for mass-energy densities of the extended particle. The Newton empty space model vs. the Cartesian material space of overlapping extended vortexes is illustrated in Figure 1. No one found and justified a mesoscopic scale for a possible transition from non-dual microscopic physics to the dual macroscopic model of Newton. Elementary material states should be considered in non-dual field terms on micro, macro and mega scales, at least in qualitative approaches, if not in quantitative ones.
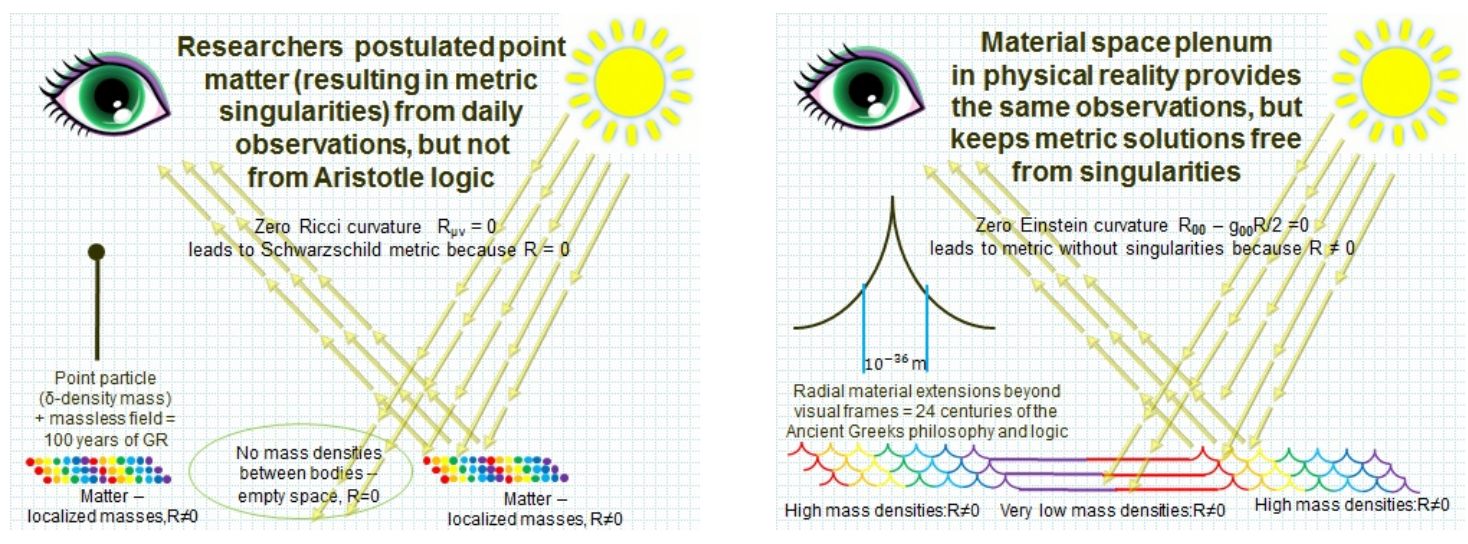

Figure 1. Newtonian particles and empty space vs. Cartesian non-dual plenum.

The purpose of this paper is to trace the balanced energy origin of extended charges in Maxwell's electrodynamics, to study strong field energy conservation and compensation for overlapping material elements in terms of complex densities, to propose non-dual field analogues of the Maxwell equations. Based on propositions of Aristotle, Descartes, Mie [9] and Einstein [10], we shall try to redesign classical electrodynamics in pure field terms (material fields without localized particles). We shall try to relate the Western science definitions to the Eastern teaching on the coexistence of corporeal (yang) and incorporeal (yin, immeasurable) worlds. Our initial idea is that the science motto 'Nullius in verba' may coherently replace the supposed empty space (between the localized charges) with the Aristotle space plenum of charged material fields. This will assist to give up the unnecessary concept of Newton's point-like particles and to modify the old building of classical electrodynamics by strong field analytical solutions without energy divergence.

Nonempty space with a continuous charge density and a density of continuous mass will have the energy meaning in our quantitative analysis. All material densities will universally obey the Yin-Yang Principle for local pairing of complementary (passive and active) energy flows. This Chinese principle stands behind the principle of equivalence of Einstein for gravitational (passive) and inertial (active) masses of mechanical bodies. We will track the same yin-yang relations for equal passive and active electric charges and their compensated energies in Maxwell's electrodynamics. The yin-yang dialectic will suggest to unify the charge with its field, as well as electricity with gravity. For this, we will use complex numbers, while all four known interactions may be unified similarly in algebra of quaternions, for example.

This article is not a polemical overview of the philosophical treasury of Descartes or the well-known neo-Cartesian approaches to nature. We are focused mainly on the mathematical energy balances for 
the introduced radial charge instead of the point particle in order to reinforce the non-empty space idea for Maxwell's electrodynamics and for Einstein's theories of relativity. The Cartesian idea of extended charges was qualitatively discussed by a number of authors who considered the Dirac delta-density as a formal limit to simplify computations in extreme fields. Our non-dual field approach works with analytical strong-field solutions to the classical field equations rather than with the delta-operator approximations. The nonlocal reading of extended electric and inertial charges may be useful for those researchers who would like to look at the world holism and Cartesian nonlocality beyond the operator procedures of quantum mechanics. Our mathematics of continuous charges is free of energy divergence, but is still a palliative model for further approximations of the very complex physical reality. Fundamental thought experiments and universal dialectical ideas should dominate the further evolution of relativistic physics and its mathematical support.

\section{Coulomb Fields within Continuous Astrocharges}

The predominant majority of people believe that electric charges and inertial masses are located within the visible frame of macroscopic bodies. Such bodies can be divided into smaller parts in accordance with everyday observations. Thus, the smallest part of a substance was introduced as a so-called corpuscle (the point particle in the indivisible limit) that is a carrier of the elementary mass and charge. Classical fields between localized particles are considered massless and chargeless. Nevertheless, the experiment is only a criterion of truth, but not the truth itself. The Newtonian pragmatism of highly-educated and well-equipped contemporary proponents of the supposed dual 'reality' (particle and field are different notions) was rejected by many thinkers. Indeed, by dividing an infinitely-extended field-energy object into parts, one should again obtain only infinite objects rather than corpuscles of limited size.

In contrast to available observations, nonempty space of continuous material flows has been recognized by philosophers not only in the Ancient East and in Ancient Greece, but also by many contemporary scholars around the world. "A coherent field theory requires that all elements be continuous... And from this requirement arises the fact that the material particle has no place as a basic concept in a field theory. Thus, even apart from the fact that it does not include gravitation, Maxwell's theory cannot be considered as a complete theory", as was stated by Einstein and Infeld [10] in 1938. Indeed, the postulated point-particle paradigm results in Coulomb divergence, which terminates classical electrodynamics as a self-consistent theory. A point source in the Maxwell-Lorentz equations may be considered as "an attempt which we have called intellectually unsatisfying", according to De Broglie [11]. Einstein also criticized his 1915 field equation for the point gravitational source: "it resembles a building with one wing built of resplendent marble and the other built of cheap wood" (translation: [12]). The Dirac delta-operator for point material densities in emptiness seems to have pushed physicists a bit away from the physical reality.

Continuously-distributed elementary charge was reasonably inferred by Mie in order to derive properties of nonlocal charges from properties of their EM fields and potentials. Regretfully, in 1912-1913 the 'Theory der Matter' [9] had not found the exact (logarithmic) potential of strong fields within nonlocal classical matter. Therefore, the promising non-empty space concept had not been proven before the mathematical era of quantum non-locality began. Formal probabilities of the delta-operator 'dice' in the Newtonian empty space arena postponed the systematic search of rigorous analytical solutions for Mie's nonlocal world of continuous particles. However, many leaders of the quantum theory appreciated the idea of the radial classical electron, for example Schwinger in the publication dedicated to Dirac [13].

The classical field equations keep the analytical solution option for a distributed elementary particle. By following Mie, we assume that it is possible to relate locally electric, $\vec{d}(x)$, and magnetic, $\vec{b}(x)$, field intensities in the Maxwell-Lorentz equations [14], 


$$
\left\{\begin{array}{l}
\operatorname{div} \vec{d}(\mathbf{x}, t)=4 \pi \rho(\mathbf{x}, t) \\
\operatorname{div} \vec{b}(\mathbf{x}, t)=0 \\
\operatorname{ccurl} \vec{b}(\mathbf{x}, t)=4 \pi \rho(\mathbf{x}, t) \vec{v}+\partial_{t} \vec{d}(\mathbf{x}, t) \\
\operatorname{ccurl} \vec{d}(\mathbf{x}, t)=-\partial_{t} \vec{b}(\mathbf{x}, t)
\end{array}\right.
$$

to charge, $\rho(\mathbf{x}, t)$, and current, $\rho(\mathbf{x}, t) \vec{v}$, densities of one extended electron. Contrary to Lorentz, the electron's charge distribution was not postulated by Mie within a microscopic spatial volume (electrons' fields were assigned by Lorentz to charge-free regions or to the supposed empty space around charges). The mathematical equation $\operatorname{div} \vec{d}(\mathbf{x}, t)=4 \pi \rho(\mathbf{x}, t)$ can be rigorously resolved under the nonempty space assumption by admitting that the elementary charge and its field coexist together in all space points $\mathbf{x}$ of the infinite Universe. In other words, we maintain the Mie (and Einstein) idea that the elementary electric (and gravitational) charge is to be inseparably integrated into its spatial field structure with instantaneous local relations.

The distributed electron holds a constant charge, $q \equiv \int \rho\left(r, r_{q}, q\right) 4 \pi r^{2} d r=$ const, associated in equilibrium with the radial electric density $\rho\left(r, r_{q}, q\right)$. The latter is defined by the spatial shaping scale $r_{q}$, which in turn depends on the integral value $q$. The analytical material density $n\left(r, r_{q}, q\right) \equiv \rho\left(r, r_{q}, q\right) / q$ (instead of the Dirac delta-density $\delta(r)$ of the point charge) should be able to possess also the inertial and gravitation charges $\sqrt{G} m_{0}$ as integrals of elementary densities. Einstein formulated the celebrated principle of equivalence for inertial and heavy masses of mechanical bodies. If this principle is a part of the general dialectics for all charges in natural phenomena, then one should extend the principle of equivalence of Einstein on (passive and active) electric charges. Following this way, the passive charge density of the extended electron $q n(x)$ should be accompanied by an equal charge density composed of Coulomb fields. In the Einstein-type terminology, the passive charge density $\rho_{p}(\mathbf{x}, t) \equiv \nabla \vec{d}(\mathbf{x}, t) / 4 \pi$ of the extended particle numerically coincides with the active charge density of the static Coulomb field:

$$
\rho_{a}(\mathbf{x}, t) \equiv[ \pm \vec{d}(\mathbf{x}, t)]^{2} /\left(4 \pi \varphi_{0}\right),
$$

where $\rho_{a}(\mathbf{x}, t) \equiv \rho_{p}(\mathbf{x}, t)=\rho(\mathbf{x}, t)$ and $q_{a} \equiv q_{p}=q$. Hereinafter, $\varphi_{o}=$ const is the universal self-potential for densities of active charges like $c^{2}$ is the self-potential of masses in the relativistic energy $m c^{2}$. Such local physical relations between the electron's continuous density and its Coulomb's field intensity can be found [15] as an exact static solution $(\vec{v}=0$ and $\vec{b}=0)$ to the Maxwell-Lorentz equations (1) for one extended charge,

$$
\left\{\begin{array}{l}
\vec{d}\left(r, r_{q}, q\right)=q \hat{\mathbf{r}} /\left[r\left(r+r_{q}\right)\right] \\
\rho\left(r, r_{q}, q\right)=q r_{q} /\left[4 \pi r^{2}\left(r+r_{q}\right)^{2}\right]=\nabla \vec{d}\left(r, r_{q}\right) / 4 \pi=r_{q} \vec{d}^{2}\left(r, r_{q}\right) /(4 \pi q)
\end{array}\right.
$$

Here, $r_{q}=$ const is the integration parameter (subject to be defined through the elementary system integral $q$ ) in the first-order differential equation div $\vec{d}=4 \pi \rho$. By following $E_{m}=m c^{2}$ for the relativistic self-energy of Einstein, we define the active electric self-energy density as $\rho_{a} \varphi_{o}$ and the elementary charge self-energy as $E_{q} \equiv \int \rho_{a} \varphi_{0} d V \equiv q_{a} \varphi_{0}$. Then, the active extended charge $q_{a} \equiv \int d V \vec{d}^{2} /\left(4 \pi \varphi_{0}\right)$ has the electric energy nature like the extended inertial mass has the mechanical (kinetic) energy nature. We assume that the new potential $\varphi_{0} \equiv q / r_{q} \Rightarrow c^{2} / \sqrt{G}$ in the solution (3) takes universally a similar ratio, $\sqrt{G} m / r_{m}=c^{2} / \sqrt{G} \equiv \varphi_{0}$, of the charge value to the shaping scale in the gravitational theory of extended mass-energies [15,16]. With such an intuitive similarity, the electric self-energy $E_{q}=q_{a} \varphi_{o}$ of the active charge $q_{a}$ should be added to the internal self-energy $E_{m}=m c^{2}$ of the active, inertial mass.

The equilibrium density (3) of one elementary extended charge $q$ depends only on one specific value, $\rho\left(r, r_{q}, q\right) \Rightarrow \rho(r, q)=q^{2} \varphi_{o} /\left[4 \pi r^{2}\left(r \varphi_{o}+q\right)^{2}\right]$. Multi-body dynamic reality will change the radial solution (3) for an isolated electron. Dynamic solutions for elementary densities at more or less generic initial conditions require specific studies that lie outside of our next goals in order to trace the Yin-Yang dialectics for pairing of active and passive energies. 
The Maxwell-Lorentz Equations (1) can be discussed as in Newtonian dual terms of electric fields and their localized currents, as well as in Cartesian non-dual terms of locally-paired material fields with continuous active/passive charge densities in the conjugated structure of active/passive energy streams. Potential self-energies of passive charge densities in their own electric/gravitational fields should compensate quantitatively active self-energies of active charge densities. Recall that the passive self-energies are infinite for point particles in the dual approach of Newton. However, the Cartesian non-dual world of extended elementary carriers is free from diverging energy integrals, and we can keep the potential self-energy. In order to reach mutual compensations of active and passive self-energies, we associate Cartesian electricity with imaginary numbers by using $\int \rho(x) d V$ $\equiv q \equiv i e \Rightarrow-i e_{0}$ for the 'negative' elementary charge of the electron. Now, we use the fundamental potential $\varphi_{0} \equiv c^{2} / \sqrt{G}=1.04 \times 10^{27} V$ for active self-energies of both inertial (real) and electric (imaginary) extended charges,

$$
E=(\sqrt{G} m+q) \varphi_{0}=i(e-i \sqrt{G} m) \frac{c^{2}}{\sqrt{G}}
$$

in order to upgrade the Einstein mechanical formula by electricity [17].

Despite that the imaginary electric charge $q=-i e_{0}$ possesses in (4) the imaginary electric energy $-i \cdot 1.04 \times 10^{24} \mathrm{KeV}$ next to $511 \mathrm{KeV}$ of the real mechanical energy, paired interactions of imaginary electric charges correspond to real Coulomb forces and to real interaction energies. Contrary to the Coulomb electric charge defined in real numbers, like the inertial mass, the imaginary charge exhibits the correct direction of the radiation self-force $2 q^{2} \ddot{\vec{v}} / 3 c^{3}$, which is proportional to $q^{2}=\left(-i e_{0}\right)^{2}<0$. Ultimately, joint continuous densities of elementary inertial and electric charges can be described in complex functions. Real integrals over spatial densities provide inertial mass-energies, while imaginary integrals correspond to non-inertial self-energies of electric charges.

\section{Superimposition of Extended Charges with Conservation and Compensation of Active/Passive Field Energies}

The strong-field potential $W(\mathbf{x})=-\varphi_{0} \ln \left[1+\sqrt{G} m /|\mathbf{x}| \varphi_{0}\right] \neq$ const within the extended gravitational/inertial charge $\sqrt{G} m$ was introduced [15] for the local action on probe bodies and for the local gravitational self-action of massive densities themselves. In this view, the inertial (active, positive, kinetic, yang) energy $\varphi_{0} \sqrt{G} m=m c^{2}>0$ of the active charge $\sqrt{G} m$ is accompanied by the negative gravitational (passive, negative, potential, yin) self-energy $\left(-m c^{2}\right)$ because of the gravitational self-action of the negative potential $W(\mathbf{x})$ on its positive mass-charge densities $\sqrt{G} \rho_{m}(\mathbf{x})=\sqrt{G} m r_{m} /\left[4 \pi \mathbf{x}^{2}\left(r_{m}+|\mathbf{x}|\right)^{2}\right], r_{m} \equiv G m / c^{2}$. Computations provide exact compensation of active (yang, measurable) and passive (yin, unmeasurable) energy integrals for every radial (elementary) carrier of inertia and gravitation,

$$
\int W(\mathbf{x}) \sqrt{G} \rho_{m}(\mathbf{x}) d^{3} x=-m c^{2} \equiv-\varphi_{0} \sqrt{G} m=-\int \varphi_{0} \sqrt{G} \rho_{m}(\mathbf{x}) d^{3} x .
$$

This integral compensation of the internal (kinetic, active, inside heat) rest-mass energy $m c^{2}$ by the negative potential energy from the gravitational self-action, $\int\left[\varphi_{0}+W(\mathbf{x})\right] \sqrt{G} \rho_{m}(\mathbf{x}) d^{3} x \equiv 0$, takes place under the integral mathematical equality $\int_{0}^{\infty} d x\left[\ln \left(1+x^{-1}\right)\right] /(1+x)^{2} \equiv \int_{0}^{\infty} d x /(1+x)^{2}$ $=1$. Similarly, passive electric densities in their strong-field logarithmic potential $W(\mathbf{x})$ should also lead to the strict compensation of the active electric energy of post-Coulomb fields. This analogy with gravitation works only for imaginary electric charges. Their kinetic-type active energy and the potential self-energy are both infinite in the point charge model. However, they are finite under the Cartesian consideration of continuous particles. Imaginary values of these paired electric energies are conjugated and exhibit the same zero sum compensation as the relativistic 'rest energy' and its negative gravitational self-energy. In practice, one may measure only active, kinetic energies, while complimentary (passive, potential) energies remain unmeasurable. For this reason, one can 
reinforce the yin-yang terminology regarding pairing of active and passive energy flows, both in the real mass gravitation and in the imaginary charge electrodynamics.

The gravity-type Poisson equation, $\nabla^{2} W=4 \pi \rho \Rightarrow \varphi_{o}^{-1}(\nabla W)^{2}$, treats the imaginary astroelectron as a non-linear field composition with respect to the radial field intensity $d(r)$ or the electron's post-Coulomb logarithmic potential $W(r)$, with $d(r)=-\partial_{r} W(r)$ and:

$$
\frac{\partial_{r}\left[r^{2} \partial_{r} W(r)\right]}{4 \pi r^{2}} \equiv \frac{\left[\partial_{r} W(r)\right]^{2}}{4 \pi \varphi_{o}}=\frac{q r_{q}}{4 \pi r^{2}\left(r+r_{q}\right)^{2}} .
$$

This non-linear equation reveals the imaginary post-Coulomb potential with the negative, gravitational sign for the continuous radial carrier of electricity,

$$
W(r)=-\frac{q}{r_{q}} \ln \left(1+\frac{r_{q}}{r}\right) \equiv-\varphi_{o} \ln \left(1+\frac{q}{\varphi_{o} r}\right) .
$$

This logarithmic potential for strong radial fields reproduces the Coulomb law on measurable scales, $\left|r_{q}\right| \ll r$, but with the negative sign like in the Newton gravitational law. In general, the integration constant $r_{q} \equiv q / \varphi_{0}$ can be a complex number, if the charge $q$ is complex or imaginary. Such a Newton-type potential of imaginary charges describes real electric forces, $q_{1}\left[-\nabla\left(-q_{2} r_{q}^{-1}\right) \ln \left(1+r_{q} r^{-1}\right)\right]=i e_{1}\left(-i e_{2}\right) \hat{\mathbf{r}} / r\left(r+r_{q}\right) \approx e_{1} e_{2} \hat{\mathbf{r}} / r^{2}$, with the mutual repulsion of like charges $e_{1}$ and $e_{2}$ and the attraction of unlike ones.

The gravitational sign in the Poisson equation for imaginary electric energies suggests to invert positive and negative imaginary densities of electric charges, $\rho \rightarrow-\rho$, in the Maxwell-Lorentz Equation (1). The conventional inversion of electric charge signs for electrons and protons does not change the electrodynamic laws of paired interactions. The electrostatic solution for charged imaginary densities in (1) with the gravitational type direction of the radial Coulomb field, $\vec{D}(\mathbf{x})=$ $-Q \hat{\mathbf{x}} /|\mathbf{x}|\left(|\mathbf{x}|+Q \varphi_{o}^{-1}\right)$, facilitates the unification of gravity and electricity on the basis of one complex energy-charge (4), $r_{Q} \varphi_{o}^{2} \equiv\left(q_{m}+i q_{e}\right) c^{2} / \sqrt{G} \Rightarrow(\sqrt{G} m+i e) c^{2} / \sqrt{G}$. The Maxwell-Lorentz Equation (1) for one massless elementary charge can be extended to the complex energy flows of one radial carrier with the complex charge density $\rho_{Q}(\mathbf{r}, t) \equiv(\sqrt{G} m+i e) n\left(\mathbf{x}, t, r_{Q}\right)=-\operatorname{div} \vec{D}(\mathbf{x}, t) / 4 \pi$,

$$
\left\{\begin{array}{l}
\operatorname{div} \vec{B}(\mathbf{x}, t) \varphi_{o}=0 \\
\operatorname{ccurl} \vec{B}(\mathbf{x}, t) \varphi_{o}=\vec{v} d i v \vec{D}(\mathbf{x}, t) \varphi_{o}+\partial_{t} \vec{D}(\mathbf{x}, t) \varphi_{o} \\
\operatorname{ccurl} \vec{D}(\mathbf{x}, t) \varphi_{o}=-\partial_{t} \vec{B}(\mathbf{x}, t) \varphi_{o} .
\end{array}\right.
$$

Here, we replaced the extended charge density $\rho_{Q}(\mathbf{r}, t)$ in the former current equations with the equivalent field flow from the local divergence. The complex gravitoelectric, $\vec{D}(x)$, and gravitomagnetic, $\vec{B}(x)$, fields of the integral elementary charge $Q=q_{m}+i q_{e}$ in (8) are model analogies of the Lorentz fields $\vec{d}(x)$ and $\vec{b}(x)$, respectively, in the basic Equation (1).

By locally pairing energy flows of the radial Newton-Coulomb fields and the densities of the continuous particle, one also unifies in (8) gravimechanical and electric self-energies $q \varphi_{o} \Rightarrow Q \varphi_{0}=$ $(\sqrt{G} m+i e) c^{2} / \sqrt{G}$. Such a double unification (particle with field and gravity with electricity) requires one (but complex) charge $q \Rightarrow Q=(\sqrt{G} m+i e)$ in the logarithmic potential (7). One can verify quantitatively that the complex (astro)charge distribution, $\rho(r)=Q n\left(r, r_{q}\right)=(\sqrt{G} m+i e) n\left(r, r_{q}\right)$ with $r_{q} \Rightarrow z_{Q}=Q \sqrt{G} / c^{2}$, generates this strong field potential under the regular integral rule for classical gravitation (and electrodynamics of imaginary charges),

$$
\begin{array}{r}
W_{Q}(r)=-\int \frac{Q n\left(r^{\prime}, z_{Q}\right) d V^{\prime}}{\left|\vec{r}-\vec{r}^{\prime}\right|}=-\int_{0}^{\infty} \int_{-\pi / 2}^{\pi / 2} \int_{0}^{2 \pi} \frac{d \phi^{\prime} \sin \theta^{\prime} d \theta^{\prime} r^{\prime 2} d r^{\prime}}{\sqrt{r^{2}+r^{\prime 2}-2 r r^{\prime} \cos \theta^{\prime}}} \frac{Q z_{Q}}{4 \pi r^{\prime 2}\left(r^{\prime}+z_{Q}\right)^{2}}= \\
-\int_{0}^{\infty} \frac{d r^{\prime} Q z_{Q}}{\left(r^{\prime}+z_{Q}\right)^{2}}\left(\frac{\left|r^{\prime}+r\right|-\left|r^{\prime}-r\right|}{2 r r^{\prime}}\right)=-\int_{r}^{\infty} \frac{Q d r^{\prime}}{z_{Q}}\left(\frac{1}{r^{\prime}}-\frac{1}{r^{\prime}+z_{Q}}\right) \equiv-\frac{Q}{z_{Q}} \ln \left(1+\frac{z_{Q}}{r}\right)=\int_{r}^{\infty} \hat{\mathbf{r}}^{\prime} \vec{D}\left(r^{\prime}\right) d r^{\prime} .
\end{array}
$$


Notice from the last relation in (9) that $W_{Q}(r)$ coincides with the complex work associated with replacements of a unit probe charge from the point $r$ to $\infty$ against the radial field $\hat{\mathbf{r}}^{\prime} \vec{D}\left(r^{\prime}\right)=D\left(r^{\prime}\right)=$ $-\partial_{r^{\prime}} W\left(r^{\prime}\right)$. The integration over $r^{\prime}$ within $0 \leq r^{\prime} \leq r$ vanishes identically in (9) in agreement with the physical meaning of potentials for a probe body.

The multibody system of complex charges $Q_{k}=\sqrt{G} m_{k}+i e_{k} \equiv \varphi_{o} z_{k} \equiv \mathcal{E}_{k} / \varphi_{o}$ with strong field electric and gravitational interactions can be described by the 'long' logarithmic potential:

$$
W_{s y s}(\mathbf{x})=-\frac{c^{2}}{\sqrt{G}} \ln \left(1+\frac{z_{1}}{\left|\mathbf{x}-\mathbf{a}_{1}\right|}+\frac{z_{2}}{\left|\mathbf{x}-\mathbf{a}_{2}\right|}+\ldots+\frac{z_{n}}{\left|\mathbf{x}-\mathbf{a}_{n}\right|}\right) \text {. }
$$

Like in the equality (6), the principle of equality universally works for net active and passive densities of overlapping complex charges in all field points of the common material space-plenum,

$$
\rho_{a}(\mathbf{x}) \equiv \frac{\left[-\nabla W_{s y s}(\mathbf{x})\right]^{2}}{4 \pi \varphi_{o}}=\frac{\nabla^{2} W_{s y s}(\mathbf{x})}{4 \pi} \equiv \rho_{p}(\mathbf{x})
$$

In spite of paired interactions of elementary extended charges, their volume energy integral demonstrates the system energy conservation law due to interference (dark, invisible) exchanges $2 A B$ in the algebra structure $(A+B)^{2}$ of overlapping energy densities,

$$
\mathcal{E}_{s y s} \equiv \int \rho_{a}(\mathbf{x}) \varphi_{o} d^{3} x=\frac{\varphi_{o}^{2}}{4 \pi} \int\left(\frac{\frac{\left(\mathbf{x}-\mathbf{a}_{1}\right) z_{1}}{\left|\mathbf{x}-\mathbf{a}_{1}\right|^{3}}+\frac{\left(\mathbf{x}-\mathbf{a}_{2}\right) z_{2}}{\left|\mathbf{x}-\mathbf{a}_{2}\right|^{3}}+\ldots+\frac{\left(\mathbf{x}-\mathbf{a}_{n}\right) z_{n}}{\left|\mathbf{x}-\mathbf{a}_{n}\right|^{3}}}{1+\frac{z_{1}}{\left|\mathbf{x}-\mathbf{a}_{1}\right|}+\frac{z_{2}}{\left|\mathbf{x}-\mathbf{a}_{2}\right|}+\ldots+\frac{z_{n}}{\left|\mathbf{x}-\mathbf{a}_{n}\right|}}\right)^{2} d^{3} x \equiv \varphi_{o}^{2} \sum_{k=1}^{n} z_{k} \equiv \sum_{k=1}^{n} \mathcal{E}_{k},
$$

again, mono-radial densities $A^{2}\left(\mathbf{x}-\mathbf{a}_{A}\right)$ and $B^{2}\left(\mathbf{x}-\mathbf{a}_{B}\right)$ can be interpreted under observations as elementary monopoles of radial energy due to the measurable structure of their Coulomb/Newton fields. It is possible to extract these mono-radial carriers from a visible substance like its parts (radial particles or monopoles). Based on the observations in practice, it can be assumed that only mono-radial elementary carriers can constitute any visible substance. Indeed, bipoles or bi-radial material densities $A\left(\mathbf{x}-\mathbf{a}_{A}\right) B\left(\mathbf{x}-\mathbf{a}_{B}\right)$ in the energy integral (12) cannot be in principle retrieved from the visible body together with one or another isolated particle. Thus, the interference (invisible, dark) energy densities of bipoles can be justified for electrodynamics and gravitation only from calculations, but not from direct observations and measurements.

Another universal law of this Cartesian physics for the system of continuous material fields is the exact compensation of post Coulomb/Newton active energies (12) by the self-action of passive charged densities from (11) in their relativistic potential (10):

$$
\begin{array}{r}
\int\left(\frac{\frac{\left(\mathbf{x}-\mathbf{a}_{1}\right) z_{1}}{\left|\mathbf{x}-\mathbf{a}_{1}\right|^{3}}+\frac{\left(\mathbf{x}-\mathbf{a}_{2}\right) z_{2}}{\left|\mathbf{x}-\mathbf{a}_{2}\right|^{3}}+\ldots+\frac{\left(\mathbf{x}-\mathbf{a}_{n}\right) z_{n}}{\left|\mathbf{x}-\mathbf{a}_{n}\right|^{3}}}{1+\frac{z_{1}}{\left|\mathbf{x}-\mathbf{a}_{1}\right|}+\frac{z_{2}}{\left|\mathbf{x}-\mathbf{a}_{2}\right|}+\ldots+\frac{z_{n}}{\left|\mathbf{x}-\mathbf{a}_{n}\right|}}\right)^{2} \frac{\varphi_{o}^{2} d^{3} x}{4 \pi} \\
-\int\left(\frac{\frac{\left(\mathbf{x}-\mathbf{a}_{1}\right) z_{1}}{\left|\mathbf{x}-\mathbf{a}_{1}\right|^{3}}+\frac{\left(\mathbf{x}-\mathbf{a}_{2}\right) z_{2}}{\left|\mathbf{x}-\mathbf{a}_{2}\right|^{3}}+\ldots+\frac{\left(\mathbf{x}-\mathbf{a}_{n}\right) z_{n}}{\left|\mathbf{x}-\mathbf{a}_{n}\right|^{3}}}{1+\frac{z_{1}}{\left|\mathbf{x}-\mathbf{a}_{1}\right|}+\frac{z_{2}}{\left|\mathbf{x}-\mathbf{a}_{2}\right|}+\ldots+\frac{z_{n}}{\left|\mathbf{x}-\mathbf{a}_{n}\right|}}\right)^{2} \ln \left(1+\sum_{k=1}^{n} \frac{z_{k}}{\left|\mathbf{x}-\mathbf{a}_{k}\right|}\right) \frac{\varphi_{o}^{2} d^{3} x}{4 \pi} \equiv 0 .
\end{array}
$$

In (10) and (11), we consider only stationary systems without magnetic fields connected with the energy currents. Therefore, we work in the local and integral balances (11) and (13), respectively, with only one system potential (10). This inferred logarithmic potential corresponds to the Einstein Principle of Equivalence for active and passive charge densities. The Principle of Equivalence is essential for our theory, which aims at strict mutual compensation of active (yang) and passive (yin) energies. If someone rejects the equivalence (11) for local densities and integral charges for some reason, it would be impossible to apply the Yin-Yang Principle for the (unbalanced) energies of such nonequivalent charges. In other words, we assumed the Einstein-type balance for densities of complex 
charges, and we found that this assumption can be justified by the yin-yang dialectics for energies of such charges.

The Cartesian elementary system, like a multi-body galaxy or one continuous electron with distributed densities, exhibits the yin-yang compensation of passive and active energies and the strict conservation of the active (measurable) fraction of these energies (as well as the conservation of the passive fraction). The mass/charge creation mechanism from 'the void nothing' (i.e., from the net zero energy) stands behind the global integral compensation (13) for yin-yang paired complex energies. The Cartesian world of extended matter is a self-consistent one in terms of continuous field energies. The latter are free from mathematical singularities and Coulomb/Newton energy divergence.

\section{Maxwell Equations Are Quantitative Identities for Paired Energy Flows}

The exact analytical solution (3) to Maxwell's equations rigorously requests the $r^{-4}$ radial astroparticle instead of the conventional point particle (or the smallest material corpuscle assumed by observers in microscopic volumes). However, rigorous solutions demand that the empty space paradigm of Newton (presumably resulting from observations and measurements) should be replaced with the non-empty space Cartesian concept to achieve a coherent theory of classical fields. Distributed elementary energies with smooth densities seem to stand behind the physical reality. The latter is available to everyone in observations of regions with high (Newtonian substance) concentrations of active energy (inside heat or kinetic degrees of freedom). Potential and self-potential energies are beyond direct measurements in experimental studies and observations.

Like the first pair of Maxwell identities, $\nabla_{\lambda} F_{\mu \nu}+\nabla_{\mu} F_{\nu \lambda}+\nabla_{\nu} F_{\lambda \mu} \equiv 0$, the second pair of Maxwell equations for currents can also be considered (in the non-empty space paradigm) as quantitative identities for the balanced four-momentum of paired energy densities,

$$
\left\{\begin{array}{l}
\varphi_{o} \nabla_{\lambda} F_{\mu v}(x)+\varphi_{o} \nabla_{\mu} F_{v \lambda}(x)+\varphi_{o} \nabla_{v} F_{\lambda \mu}(x) \equiv 0 \\
{\left[\delta_{\lambda}^{\mu}-u^{\mu}(x) u_{\lambda}(x)\right] \varphi_{o} \nabla_{v} F^{\lambda v}(x) \equiv \varphi_{o} S^{\mu}(x) .}
\end{array}\right.
$$

Here, instead of $\nabla_{v} F^{\mu v}(x) \equiv-4 \pi j^{\mu}(x) / c$ for classical electrodynamics of real electric charges, we use the opposite, gravitational sign to relate tensor fields and vector flows of electromechanical continuous charges $Q=q_{m}+i q_{e}$ in the following identical relations for real gravity and imaginary electricity:

$$
\left\{\begin{array}{l}
\nabla_{v} F^{\mu v}(x) \equiv+4 \pi j^{\mu}(x) / c \equiv 4 \pi \rho(x) u^{\mu}(x)+S^{\mu}(x) \\
u_{\mu}(x) S^{\mu}(x) \equiv 0, \quad u_{\mu}(x) \nabla_{v} F^{\mu v}(x) \equiv 4 \pi \rho(x) \\
\nabla_{v} F^{\mu v}(x) \equiv u^{\mu}(x) u_{\lambda}(x) \nabla_{v} F^{\lambda v}(x)+S^{\mu}(x)
\end{array}\right.
$$

The four-acceleration balance in the second identity in (14) requires $S^{\mu}=0$ for non-accelerated energy currents, like in (1). The requirement $S^{\mu}=0$ along the four-acceleration axis, which is normal to the four-velocity $u^{\mu}$, means the absence of radiation or heat energy flows, circular spins and turbulence motions in the steady laminar four-flow of energy $\varphi_{o} \rho(x) u^{\mu}(x)$. The material reality of non-empty three-space is nothing but paired energy flows in all spatial points of the Universe, filled continuously with equal densities of active and passive continuous charges, but opposite integrals of elementary energies. Again, equal local densities of complex charges (11) have only the integral compensation (13) for paired energy densities. The latter are distributed differently over points of the system material space. Basing on the mathematical compensation of the complex energies (13), one can relate their real parts to the inertial (mechanical) energy content of the elementary system, while their imaginary parts to the elementary electric charge of this system (the electron or a galaxy, for example). There is not much benefit from Newtonian scalar mass in the pure field developments of inertial Cartesian densities of warm material space with the inside heat variable [18]. 
If one considers the paired electric currents through the coupled field flows of active and passive energies in the Maxwell-Lorentz theory for the extended electron, then the Lagrange variations in nonempty space physics must be different from the textbook variation procedures for the empty space system of spatially-separated particles and fields. Indeed, the current Maxwell equations were derived in the dual action approach to separated fields and particles (which can be varied and fixed independently, like $\delta\left[A_{\mu} j^{\mu}\right]=j^{\mu} \delta A_{\mu}, j^{\mu}=$ const). In the Cartesian field approach to extended matter-energy, self-energy variations of locally-paired flows are coupled inseparably. The variation $\delta\left(A_{\mu} j^{\mu}\right)$ is equal to the sum $j^{\mu} \delta A_{\mu}+A_{\mu} \delta j^{\mu}$ that doubles the similar result in dual Newtonian physics,

$$
\delta\left(c A_{\mu} \nabla_{v} F^{\mu v} / 4 \pi\right)=(c / 4 \pi)\left[\delta A_{\mu} \nabla_{v} F^{\mu v}+A^{\mu} \nabla^{v}\left(\partial_{\mu} \delta A_{v}-\partial_{v} \delta A_{\mu}\right)\right] \Rightarrow(c / 2 \pi) \nabla_{v} F^{\mu v} \delta A_{\mu}=2 j^{\mu} \delta A_{\mu} .
$$

Thus, the pure field Lagrangian for paired passive and active continuous charges should possess the double field density, $2 \times F_{\mu v} F^{\mu v} / 16 \pi$, of Cartesian material extensions,

$$
S_{C a r}=-\frac{1}{c} \int d \Omega\left[\frac{1}{c} A_{\mu j} j^{\mu}-\frac{1}{8 \pi} F_{\mu v} F^{\mu v}\right],
$$

compared to the empty space alternative with spatially-separated fields and charges. The simultaneous variations in (17) with respect to both potentials $A_{\mu}$ and currents $j^{\mu}$ result in the Maxwell-type identities (14) and (15) for paired EM energy flows. These identities comply with the yin-yang dialectical approach to nature.

It is worth noting that the Cartesian action integral vanishes for the Lagrange trajectories $4 \pi j^{\mu} / c=$ $\nabla_{v} F^{\mu v}$ at $F^{\mu \nu}=-F^{v \mu}$ in (17):

$$
S_{C a r} \Rightarrow-\int \frac{d \Omega}{8 \pi c}\left[2 A_{\mu} \nabla_{v} F^{\mu v}-\left(\nabla_{\mu} A_{v}-\nabla_{v} A_{\mu}\right) F^{\mu v}\right]=-\oint \frac{d S_{v}}{4 \pi c} A_{\mu} F^{\mu v} \Rightarrow 0 .
$$

The integral nullification of the scalar Lagrange density in (18) can be related to Einstein's general covariance of metric gravitation and to the compensation (13) for active-passive energies. In other words, the action nullification on the Lagrange variational trajectory is the expected result for the non-dual physics of locally-paired energy flows. We recall that the conventional current + field action in dual physics, where $\mathbf{d}^{2} / 8 \pi$ is instead of $\mathbf{d}^{2} / 4 \pi$ in (2) and $F_{\mu v} F^{\mu v} / 16 \pi$ instead of $F_{\mu v} F^{\mu v} / 8 \pi$ in (17), does not match such a Lagrange path nullification. This obvious mathematical flaw is the penalty for using an empty space model with its misinterpretation of continuous material reality. Illogical changes in magnetic fields at fixed currents that locally and identically generate these fields, including any changes of them, have been considered by many physicists as one of the unsolved problems of Classical Electrodynamics.

\section{Discussion}

So far, there is no direct experimental evidence in favor or against of non-empty space. Today, any attempts to criticize the empty space paradigm of Newton for gravitation (resulting in unphysical black holes) are still considered to be speculations. There is no mathematical error in the Schwarzschild metric solution. Its 'curvature of the emptiness' does quantitatively describe the measured post-Newtonian corrections in weak fields. So what? The 1936 conflict of Einstein with Physical Review regarding the GR coordinate singularities revealed 'a clear understanding as to why the Schwarzschild singularities do not exist in physical reality' [19]. Cartesian non-empty space assumes a non-dual analog of the Einstein equation. There will be no place for the empty-space Schwarzschild solution in this pure field physics with the smooth metric invariant.

In contrast to the non-dual physics of field energies with only one philosophical category for material reality, the dual physics of particles and their remote fields is constantly discussing the need or lack of need of new categories, like aether, extra dimensions of the unphysical empty space, etc. The non-dual Einstein-Infeld physics identified matter, including aether, with inertial fields 
of distributed warm energy. The scalar density of the nonempty space has only one mathematical pretender in the metric theory. This is the inhomogeneous Ricci scalar. Therefore, material aether and matter-extension of fine inhomogeneous densities are the same notion in the pure field physics of Einstein and Infeld.

The area of main concentrations of energy within the infinite radial electron will always have scales of spatial shaping that are much less than the instrumental record of measured space inhomogeneity (now $10^{-19} \mathrm{~m}$ ). Indeed, half of the electron charge belongs to the sphere of extremely small radius $r_{e} \equiv\left|\sqrt{G} m_{o}+i e\right| \varphi_{o}=1.38 \times 10^{-36} \mathrm{~m}$. Another half of this astroelectron is distributed over micro, macro and mega scales. The extended astroelectron will always be found in the laboratory as an energy point under inelastic exchanges with the screen. For example, no one measured the self-consistent densities of the superfluid Cooper pair, but this does not reduce the coupled electrons to the sum of two points.

Each extended astroelectron fills the entire Universe by the active inertial and electric energy $(m \sqrt{G}+i e) c^{2} / \sqrt{G}$. This nonlocal energy keeps coherent relations between its own elementary densities simultaneously over all spatial coordinates. Photons can be regarded as wave excitations of nonlocal astroelectrons, but not as independent particles. The exchange of continuous wave excitation could be an instantaneous world process in the theory of quantized states of astroelectrons. Indeed, there is no intermediate position between two quantum states of elementary matter. The speed of light is limited by the speed of information exchange between the centers of radial astroelectrons, but not by instantaneous exchanges of elementary electromagnetic waves between self-coherent energy structures of continuous astroparticles.

It is difficult to understand why modern relativists are still working with the gravity of point (in question) masses with the corresponding metric singularities, if the non-local world of physical events has already been proven. Why are gravitation experts still calling Einstein 'a reluctant father of black holes', but not as a science opponent of unphysical metric holes? Again, Einstein has rejected Schwarzschild singularities with reasonable scientific arguments [19]. Furthermore, he very clearly explained together with Infeld that [10]: "From the relativity theory we know that matter represents vast stores of energy and that energy represents matter. We cannot, in this way, distinguish qualitatively between matter and field, since the distinction between mass and energy is not a qualitative one. By far the greatest part of energy is concentrated in matter; but the field surrounding the particle also represents energy, though in an incomparably smaller quantity. We could therefore say: Matter is where the concentration of energy is great, field where the concentration of energy is small. But if this is the case, then the difference between matter and field is a quantitative rather than a qualitative one. There is no sense in regarding matter and field as two qualities quite different from each other. We cannot imagine a definite surface separating distinctly field and matter. But the division into matter and field is, after the recognition of the equivalence of mass and energy, something artificial and not clearly defined. Could we not reject the concept of matter and build a pure field physics? What impresses our senses as matter is really a great concentration of energy into a comparatively small space". And also "We could regard matter as the regions in space where the field is extremely strong. In this way a new philosophical background could be created. Its final aim would be the explanation of all events in nature by structure laws valid always and everywhere. A thrown stone is, from this point of view, a changing field, where the states of greatest field intensity travel through space with the velocity of the stone. There would be no place, in our new physics, for both field and matter, as fields would be the only reality. This new view is suggested by the great achievements of field physics, by our success in expressing the laws of electricity, magnetism, gravitation in the form of structure laws, and finally by the equivalence of mass and energy. Our ultimate problem would be to modify our field laws in such a way that they would not break down for regions in which the energy is enormously concentrated" [10].

Einstein's non-dual way for unification of the continuous mass and its gravitational field works equally well to describe the continuous electric charge in terms of Maxwell-type classical fields. 
Again, the scale $e_{0} \sqrt{G} / c^{2}=1.38 \times 10^{-36} \mathrm{~m}$ for the main densities of the radial electron is even less than the Planck's length, $l_{p} \equiv \sqrt{\hbar c}\left(\sqrt{G} / c^{2}\right)=1.62 \times 10^{-35} \mathrm{~m}$. Such extremely small scales for elementary matter concentrations can explain the success of the $\delta$-operator modeling of the nonlocal energy carrier. However, formal interactions of point-like concentrations of charge with external electric fields is conceptually incorrect for real continuous particles because of their direct and global superimposition in the nonlocal Universe. Despite the 'apparent observation' of empty space in practice, the Dirac delta-density should be used only for engineering calculations, but not for conceptual approaches to reality of continuously charged fields. Indeed, the well-performed measurements of electric fields associated with moving charges "support the idea of a Coulomb field carried rigidly by the electron beam" $[20,21]$. These experiments confirm directly the nonempty space solution (3) where the elementary charge is continuously distributed over the radial Coulomb field. Similar nonempty space solutions can be derived in General Relativity for the extended mass [15].

In fact, General Relativity was tested only in weak fields. The model of Newtonian point-like particles can quantitatively describe both classical dynamics in Newtonian fields and small post-Newtonian corrections of the first order in empty (but curved) space. Such probes may only state that Newton and Schwarzschild can describe quantitatively the weak field approximation of reality. At the same time, nonempty (but flat) space physics also predicts [16] the measured post-Newtonian corrections in the main gravitational tests. Actually, the Descartes and Einstein-Infeld approaches are right for both weak and strong material fields. The simplified Newtonian interpretation of reality through distant massive peculiarities leads to the vulgarization of Einstein's thermomechanics of inertial energy densities with the inside relativistic heat. Cartesian physics is free from peculiarities and the black hole notion. Quantum mechanics has already justified the extension of elementary matter over all spatial scales. Today, the turning point for each researcher is whether to follow Einstein's, Descartes's and Aristotle's descriptions of the non-empty space mechanics and gravitation, or to remain adherent to the empty space paradigm of Newton.

The Cartesian world alternative in testable mathematical predictions is quite significant for all areas of natural sciences, both fundamental and applied. Contrary to the empty Newtonian space with localized matter, like in the contemporary textbooks on mechanics and gravitation, the nonempty Cartesian space of continuously-distributed matter has a real chance for the ontological convergence of classical and quantum physics in the near future. Various applications of material space begin with the Earth sciences, biology, medicine, etc., and end with astrophysics of galaxies and the continuous microcosm of one extending astroparticle.

The radial distribution of the elementary carrier of mechanical and electrical complex energies (4) is a first step toward unification of nonlocal classical and quantum matter. The half-charge shaping scale $r_{e} \equiv e_{o} / \varphi_{0}=1.38 \times 10^{-36} \mathrm{~m}$ and the minimal quantization radius $r_{h}=\hbar c /\left(e_{o} \varphi_{0}\right)=1.89 \times 10^{-34} \mathrm{~m}$ of the Bohr-Sommerfeld loop can shed new light on the physical origin of the Sommerfeld (fine structure) constant, $\alpha \equiv r_{e} / r_{h}=1 / 137$, and on the physical meaning of the Plank length, $\left(\hbar c / \varphi_{0}\right)^{1 / 2} \equiv\left(r_{e} r_{h}\right)^{1 / 2}=$ $1.62 \times 10^{-35} \mathrm{~m}$, directly from the Maxwell theory. All natural sciences, guided by the yin-yang balance of active and passive complex energies in compensated flows, can consistently describe the visible world of inertial bodies through warmed spatial densities of the non-local Universe and the world holism of all extended elements. In closing, the author encourages researchers to come back to the Cartesian matter-extension and the Chinese dialectics for better evolution of contemporary relativistic physics and related disciplines.

Conflicts of Interest: The author declares no conflict of interest.

\section{References}

1. Garber, D. Descartes's Metaphysical Physics; University of Chicago Press: Chicago, IL, USA, 1992.

2. Cottingham, J. Descartes's Physics. In The Cambridge Companion to Descartes; Cambridge University Press: Cambridge, UK, 1992; pp. 286-334. 
3. Jullien, V. Oeuvres de Descartes; Adam, C., Tannery, P., Eds.; Presses Universitaires de France: Paris, France, 1996; Volume 11.

4. Descartes, R. The Philosophical Writings of Descartes; Cottingham, J., Stoothoff, R., Murdoch, D., Kenny, A., Eds.; Cambridge University Press: Cambridge, UK, 1984; Volume 3.

5. Capra, F. The Tao of Physics; Shambhala Publications: Boston, MA, USA, 1971.

6. Young, G.M. The Russian Cosmists: The Esoteric Futurism of Nikolai Fedorov and His Followers; Oxford University Press: Oxford, UK, 2012.

7. Popescu, S. Nonlocality beyond quantum mechanics. Nat. Phys. 2014, 10, 264-270. [CrossRef]

8. Smuts, J.C. Holism and Evolution, 2nd ed.; Macmillian and Co.: London, UK, 1927.

9. Mie, G. Grundlagen einer Theorie der Materie. Ann. Phys. 1912, 37, 511-534. [CrossRef]

10. Einstein, A.; Infeld, L. The Evolution of Physics; Cambridge Press: Cambridge, UK, 1938.

11. De Broglie, L. New Perspectives in Physics; Basic Books: New York, NY, USA, 1962.

12. Tonnelat, M.-A. The Principles of Electromagnetic Theory and Relativity; Riedel Publishing Co.: Dordrecht, The Netherlands, 1966.

13. Schwinger, J. Electromagnetic mass revisited. In Quantum, Space and Time-The Quest Continues; Barut, A.O., Van der Merwe, A., Vigier, J.-P., Eds.; Cambridge University Press: Cambridge, UK, 1984; pp. 620-630.

14. Lorentz, H.A. The Theory of Electrons and Its Applications to the Phenomena of Light and Radiant Heat; G.E. Stechert Company: Leipzig, Germany, 1916.

15. Bulyzhenkov, I.E. Einstein's Gravitation for Machian Relativism of Nonlocal Energy-Charges. Int. J. Theor. Phys. 2008, 47, 1261-1269. [CrossRef]

16. Bulyzhenkov, I.E. Geometrization of Radial Particles in Non-Empty Space Complies with Tests of General Relativity. J. Mod. Phys. 2012, 3, 1465-1478. [CrossRef]

17. Bulyzhenkov, I.E. Complex charge densities unify particles with fields and gravitation with electricity. Bull. Lebedev Phys. Inst. 2016, 43, 138-142. [CrossRef]

18. Bulyzhenkov, I.E. Gravitational attraction until relativistic equipartition of internal and translational kinetic energies. Astrophys. Space Sci. 2008, 363, 39. [CrossRef]

19. Einstein, A. On a Stationary System with Spherical Symmetry Consisting of Many Gravitating Masses. Ann. Math. 1939, 40, 922-936. [CrossRef]

20. De Sangro, R.; Finocchiaro, G.; Patteri, P.; Piccolo, M.; Pizzella, G. Measuring propagation speed of Coulomb fields. Eur. Phys. J. 2015, 75, 137. [CrossRef]

21. De Sangro, R.; Finocchiaro, G.; Patteri, P.; Piccolo, M.; Pizzella, G. Why the interpretation of "Measuring propagation speed of Coulomb fields stands". Eur. Phys. J. 2017, 77, 75. [CrossRef] 